\title{
Periodic synoviosis (intermittent hydrarthrosis) with observations and studies on a patient
}

\author{
Hobart A. Reimann \\ M.D. \\ The Department of Medicine, Hahnemann Medical College and Hospital, \\ Philadelphia, Pennsylvania 19102
}

\begin{abstract}
Summary
More than 200 instances of periodic synoviosis have been recorded. It is related to periodic oedema (hereditary angioedema). Various autonomic disturbances accompany episodes. Confusion with rheumatoid arthritis persists. Theoretically an inherent rhythm or feedback mechanism operates as the cause.

The disorder in several observed patients has persisted for decades, worsened or abated. In one instance, contraceptive medication failed to suppress episodes in contrast to the effect of pregnancy in some patients. E-aminocaproic acid disturbed the rhythm and gave mild relief. A few episodes were suppressed after two surgical operations. The inhibitor of $\mathrm{Cl}$ esterase in one patient lacked as much as it does in periodic oedema.
\end{abstract}

Periodic synoviosis, a heritable, non-inflammatory, afebrile malady begins after puberty. Episodes of pain or swelling or both affect the knees and occasionally other joints. Episodes last several days alternant with symptomless intervals for decades often with semi-monthly (circabiseptan) precision. Accordingly, the names hydrarthrose périodique and Kalendarsymptome arose. Because the involved synovial membrane is not inflamed, the term periodic synoviosis is apropos. Several reviews summarized knowledge of the subject up to 1964 (Garrod, 1909; Miller and Lewin, 1924; Weiner and Ghormley, 1956; Reimann, 1963a).

Articular deformity does not ensue, nor is the general health impaired in the intervals between episodes. Various autonomic disturbances often appear during episodes. Aside from suppression of episodes in some victims during pregnancy, nothing thus far gives significant relief. To account for the disorder theoretically, either an inherent rhythm or a feedback mechanism (Morley, 1970) excites 'bioclocks' in the hypothalamus or in the synovial membrane (Richter, 1960). These 'Zeitgebers' provoke sudden accumulation of plasma in the lining and spaces of joints, tendon and ligament sheaths.
Fluid resorbs after a day or more. The recent demonstration of a biochemical anomaly similar to that found in periodic oedema (hereditary angioedema, Quincke's oedema), gives a clue to a causal factor and to a possible therapeutic approach.

Systemic involvement. Disturbances of tissues, glands and organs often accompany the episodes. Polyuria, headache, hemicrania, vomiting (Garrod, 1909), parotomegaly with sialorrhea or xerostomia, lacrimation, sweating, thoracic oppression (Schlesinger, 1926), purpura (Reimann and Angelides, 1951), urticaria, oedema and pleural effusion (Frank, 1972) may concur. In one instance after 15 years of episodes, hyperglobulinaemia ended in fatal amyloidosis (Ask-Upmark, 1963). In another patient, an electroencephalogram was abnormal. Various discomforts accompany the episodes of a person whose course and investigation are described below. Most of the extra-articular features mentioned represent autonomic neurovascular reactions probably of central origin like those in other disparate periodic disorders (Reimann, 1963a).

Relation to periodic oedema. Since 1885 several observers related periodic synoviosis to periodic oedema. According to Garrod "one can hardly avoid the conclusion that ... hydrarthrosis and oedema of the lips and eyelids were part and parcel of the same morbid cycle'. Some members of afflicted families had the two entities in synchronous, or alternant, or separate rhythm. In two instances, dermal oedema replaced synoviosis in the same tempo. Plasma infiltrates the skin in one disorder and the synovial sheaths and sacs in the other. The lack of $\mathrm{Cl}$ esterase inhibitor in one patient with periodic arthrosis (Reimann, Coppola and Villegas, 1970) was as great as in periodic oedema (Donaldson and Evans, 1963; Austin and Sheffer, 1965). That feature and a subnormal inhibitor in an unaffected brother, suggesting a familial trait, adds support to the relationship.

Relation to other arthritidies. Several patients described as having palindromic rheumatism had the onset after puberty, precise periodicity of episodes, 
no articular deformity and other features similar to periodic arthrosis. Parkes-Weber (1946), Wassmann (1950) and I (1963a, pp. 48 and 117) regarded these as more likely to be examples of the latter.

According to some authorities (Ragan, 1966; Robinson, 1971), periodic synoviosis may te an initial stage of rheumatoid arthritis. Yet, the synovial membrane alone is affected by non-inflammatory oedema. In rare instances, permanent joint deformity preceded or ensued in victims of periodic synoviosis probably as an unusal sequel or as coincidental rheumatoid arthritis (Garrod, 1909; Frenkel-Tissot, 1916). In contrast to periodic synoviosis, rheumatoid arthritis affects small joints in a deforming inflammatory process. There are fever, leukocytosis and hyperglobulinemia. The rheumatoid factor is present, the inhibitor of $\mathrm{Cl}$ esterase is increased (Donaldson, 1966) and heredity is not a dominant factor.

\section{Present studies}

After my 1963 review (Reimann, 1963a) I followed the subsequent course of three victims described therein and made special studies of one of them. Unpublished reports of three others came by letter. All six are able to carry on their usual activity after inurement to years of periodic distress. The affliction lessened in two, is unchanged in two and worse in two, but without deformity in any.

New cases. Painful swelling of a knee at irregular intervals followed a second pregnancy in Dr E. A. Jenner's patient in England. After the third pregnancy, episodes lasting several days have come regularly at 10-day intervals for 2 years. An attack of influenza suppressed one episode. Synovectomy gave no relief. A grandmother has coxalgia at unspecified intervals.

Dr W. B. Bean's patient, a woman in Iowa aged 57 years, has had periodic synoviosis of both knees for 40 years. Of late, pain also involves the right hip joint. The latex test gave a normal result.

A physician, aged 45 , in Washington State, has had the disorder that began abruptly 18 years ago. An aura of irritability precedes episodes affecting the right knee only. They lasted 5 days with about 9 days of freedom in a 13 or 14 day rhythm for 4 years. After a 9-year remission, the episodes reappeared in similar rhythm for 18 months followed by another remission for 6 months. They now recur as usual but do not incapacitate him. His father had similar arthrosis in his youth.

Previously reported cases. $\mathrm{Mr} \mathrm{V}$. S. in Paris has had periodic synoviosis of the left knee and ankle for 60 years. Pain worsened and now affects the left hip joint in unison every 11 days. His sister and two cousins have a similar disorder (p. 114, Reimann, 1963a).
Since puberty, Miss M. A. P. (p. 120, Reimann, 1963a) in Massachusetts, aged 76 years, has had 5-day swelling and pain in her right knee at 12-day intervals for 60 years. The left knee participated later. Several months' to years' long remissions ensued. Episodes ceased during an attack of varicella-zoster. In late years, discomfort lessened and the tempo lengthened to 13 days. The proximal joint of the right index finger now swells synchronnusly.

I have observed patient C. R. since 1944. Then, at age 27 , the right biceps tendon contracted for about $12 \mathrm{hr}$ limiting extension of the elbow to $150^{\circ}$, with aching but no swelling. This recurred every 13 days. After 3 months the left knee, and 2 years later, the right one began to swell painfully almost in unison (Case 5, Reimann, 1948b). The same sequence of events was observed by Perrin in 1878. In 1956, two expected episodes failed to occur after a duodenal haemorrhage, partial gastrectomy and transfusions of 8 units of blood. The usual tempo then resumed. After a minor surgical operation under local anaesthesia in 1971 followed by fever of $38.4^{\circ} \mathrm{C}$ for 1 day, the next expected episode did not happen.

Over the years, unrelated to the menopause genual involvement worsened and is accompanied by malaise and fatigue. Pain often spreads from the knees to the calf muscles and last longer. The elbovo stiffness recurs at intervals of 15-50 days; the knees swell fortnightly. About $1 \mathrm{~kg}$ of water is retained during episodes. Occasionally, both hands are oedematous. In addition, several hours of twitching and tightening precedes pain in both inguinal ligaments often severe enough to oblige a stooped walking posture. This occurs in a separate 12-day rhythm. Figure 1 portrays events as they occur. Freedom from discomfort somewhere is rare.

Contraceptive therapy. The suppression of periodic synoviosis and of other periodic disorders during pregnancy in some women suggested experimental contraceptive therapy. Although the endocrine effects are similar, methoxyprogesterone $10 \mathrm{mg}$ and ethinyl estradiol $0.05 \mathrm{mg}$ taken daily for 54 days (post-menopausal) only increased the severity of episodes. During 3 weeks, therapy with $1.25 \mathrm{mg}$ daily of premarin to control 'hot flashes' intensified the arthral pains.

Epsilon aminocaproic acid (Amicar) meliorated episodes of periodic oedema in some patients (Lundh, 1968) without affecting the complement components (Champion and Lachmann, 1969; Frank et al., 1972). The drug diffuses into joint capsules where it remains for about $16 \mathrm{hr}$ (Ahlberg, 1970). Beginning a day before a predicted episode, $6 \mathrm{~g}$ of the drug were given daily. Left knee swelling was delayed 8 days, but resumed its regular tempo. Painless, mild 2-day swelling of the right knee came 


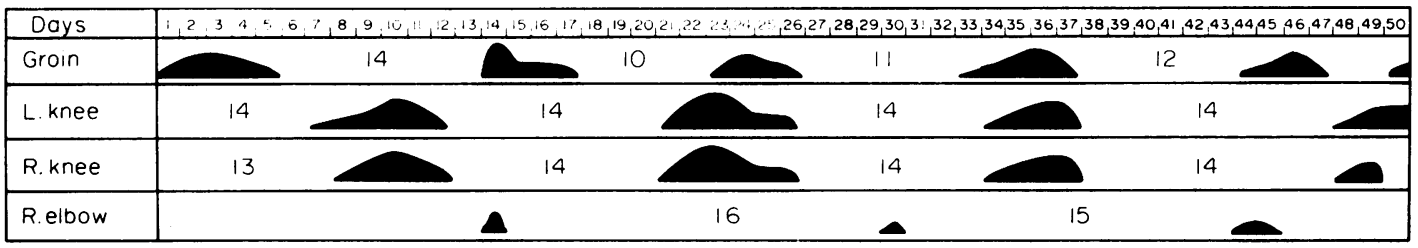

FIG. 1. Portion of an 8-month record. Groin involvement is irregular at 10- to 14-day intervals unrelated to knee episodes. Synchronized episodes of the knees occur at 14-day intervals. Elbow is affected mildly at long irregular intervals in contrast with its regular 14-day behaviour in 1963 (Fig. 34, p. 133, Reimann, 1963a). Numbers indicate onsets of involvements in days, and the shading, the degree of discomfort.

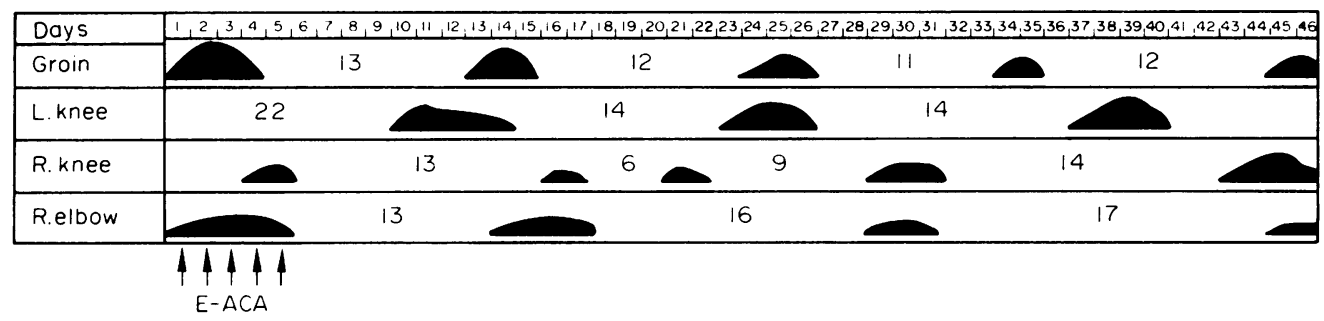

FIG. 2. Influence of E-aminocaproic acid. Groin and elbow involvement is unaffected. First episodes of the knees are delayed 8 and 3 days and the rhythms desynchronized. Four episodes of painless swelling of the right knee at 13-, 6- and 9-day intervals before resuming the basic rhythm.

3 days late. The next three episodes were short and painless, but came out-of-rhythm at 13,6 , and 9 days. Thus, for the first time in 28 years, therapy in one trial meliorated and desynchronized genual involvement. The rhythm and severity of the groin pain and elbow stiffness were unaffected. Figure 2 illustrates the events. Evidently, the drug did not affect tendon and ligament sheaths. Therapy stopped after 5 days because of intense conjunctival suffusion.

Colchicine in dosage of $0.65 \mathrm{mg}$, three times daily for 10 days prior to and overlapping a predicted episode gave no beneficial effect. Diarrhoea and nausea ended therapy.

Complement defects. As described elsewhere (Reimann, Coppola and Villegas, 1970) during symptomless times between four episodes, her serum inhibitor of $\mathrm{Cl}$ esterase measured about $2 \mathrm{u} / \mathrm{ml}$ (normal $5.6 \mathrm{u}$ ) and the whole serum complement (C) activity was $31 \mathrm{u} / \mathrm{ml}$ (normal 45-80 u). Greater reductions of inhibitor from 0.8 to $1 / 6 \mathrm{u} / \mathrm{ml}$ and of whole $\mathrm{C}$ activity from 14 to $16 \mathrm{u} / \mathrm{ml}$ characterized four episodes. Her unaffected brother's inhibitor was subnormal $(4 \mathrm{u})$ and his whole $\mathrm{C}$ activity was low at $29 \mathrm{u}$. The amounts were normal in her unaffected mother.

\section{Discussion}

The course of periodic synoviosis in five patients illustrates the uncertainty of prognosis. It may meliorate, cease or worsen over the years without permanent deformity. Various autonomic disturbances occur and a relationship to periodic oedema is evident.

In regard to observations in the case of patient C. R., the question arises whether the suppression of two episodes in 1956 resulted from shock of the surgical event, or from depletion of $\mathrm{Cl}$ esterase by haemorrhage, or from the injection of normal $\mathrm{Cl}$ esterase inhibitor in transfused blood. That the latter happened was suggested by a report wherein infusion of normal plasma containing the inhibitor was said to suppress episodes of periodic oedema (Pickering, 1969). According to a letter from Dr Virginia Donaldson, the procedure worsened the disorder at times. In 1971, the minor surgical operation followed by fever suppressed one episode.

The failure of contraceptive medication to influence the course may be partly explained since pregnancy itself does not always suppress episodes of any periodic disorder. The hormones may be of therapeutic value for women in whom gestation had been or would be suppressive. Paradoxically, if the inhibitor diminishes during pregnancy (Donaldson, 1966) and if $\mathrm{Cl}$ esterase increases, one would not expect episodes to be suppressed. Some other mechanism is involved.

E-aminocaproic acid suppressed periodic oedema without altering the defective complement components. Some patients were helped, others not (Champion and Lachmann, 1969; Frank et al., 
1972). In patient C. R., the drug during one trial de-synchronized the genual rhythm. The right knee swelled mildly and painlessly in three episodes at shorter irregular intervals before resuming the usual severity and rhythm. To be successful, any replacement or suppressant therapy must either be continuous or timed before each expected episode and free from prohibitive side-effects.

The great deficiency of $\mathrm{Cl}$ esterase inhibitor and whole serum complement, and the subnormal amounts in C.R.'s brother probably indicate a familial trait. If the inhibitor is involved in the pathogenesis of periodic oedema (Rosen and Austin, 1969), it also may be in periodic arthrosis. Whether the complemental abnormalities are causal or incidental is uncertain. Demonstration of the inhibitor lack or reduction of $\mathrm{C} 2$ and $\mathrm{C} 4$ components (Austin and Sheffer, 1965) is useful in diagnosis, and for the discovery of inapparently affected genetic relatives.

\section{References}

Ahlberg, A. (1970) Diffusion of epsilon aminocaproic acid to the joints. Proceedings Society of Experimental Biology and Medicine, 137, 988.

Ask-UPMARK, E. (1963) Further observations on periodic disorders. Acta Medica Scandinavica, 173, 115.

Austin, K.F. \& ShefFer, A.L. (1965) Detection of hereditary angioneurotic edema by demonstration of a reduction in the second component of human complement. New England Journal of Medicine, 272, 647.

Champion, R.H. \& LachmanN, P.J. (1969) Hereditary angioedema. Treatment with E-aminocaproic acid. British Journal of Dermatology, 81, 763.

Donaldson, V.H. (1966) Serum inhibitor of $\mathrm{Cl}$ esterase in health and disease. Journal of Laboratory and Clinical Medicine, 68, 369.

Donaldson, V.H. \& Evans, R.R. (1963) A biochemical abnormality in hereditary angioneurotic edema. Absence of serum inhibitor of $\mathrm{Cl}$ esterase. American Journal of Medicine, 35, 37.

Frank, N.M., Sergent, J.S., Kane, M.A. \& Alling, D.W. (1972) Epsilon aminocaproic acid therapy of hereditary angioneurotic edema. New England Journal of Medicine, 286, 808.
Frenkel-Tissot, H.C. (1916) Familiärer Hydrops Intermittens und Purin Stoffwechsel. Zeitschrift für Experimental Pathologie und Therapie, 18, 118.

GARROD, A. (1909-1910) Concerning intermittent hydrarthrosis. Quarterly Journal of Medicine, 3, 207.

Lundh, B., Laurell, A., WetTerQvist, H., White, T. \& Granerus, G. (1968) A case of hereditary angioneurotic edema treated with epsilon aminocaprin. Clinical and Experimental Immunology, 3, 733.

Miller, J.L. \& LewiN, P. (1924) Evidence of the anaphylactic character of intermittent hydrarthrosis. Journal of the American Medical Association, 82, 1177.

Morley, A.A. (1970) Periodic diseases, physiological rhythms and feedback control-a hypothesis. Australasian Annals of Medicine, 19, 244.

Pickering, R.J. et al. (1969) Replacement therapy in hereditary angioedema. Successful treatment of two patients with fresh frozen plasma. Lancet, i, 326.

Ragan, C. (1966) Intermittent Hydrarthrosis in Arthritis and Allied Conditions (Ed. by J. L. Hollander). Lea and Febiger, Philadelphia, p. 755.

Reimann, H.A. (1963a) Periodic Arthrosis in Periodic Diseases. F. A. Davis Co., Philadelphia, and Blackwell Scientific Publications, Oxford, p. 111.

ReimanN, H.A. (1948b) Periodic disease. A probable syndrome including periodic fever, benign paroxismal peritonitis, cyclic neutropenia and intermittent arthralgia. Journal of the American Medical Association, 136, 239.

Reimann, H.A. \& Angelides, A. (1951) Periodic arthralgia in twenty-three members of five generations of a family. Journal of the American Medical Association, 146, 713.

ReimanN, H.A., Coppola, E.D. \& Villegas, G.R. (1970) Serum complement defects in periodic diseases. Annals of Internal Medicine, 73, 737.

RichTER, C.P. (1960) Bioclocks in medicine and psychiatry Proceedings of the National Academy of Science U.S.A. 46, 1506.

RoBINson, W.D. (1971) Intermittent hydrarthrosis. CecilLoeb Textbook of Medicine. W. B. Saunders Co., Philadelphia and London, 13th Edition, p. 1899.

Rosen, F. \& Austin, K.F. (1969) The neurotic edema, hereditary angioedema. Editorials, New England Journal of Medicine, 280, 1356.

SCHLESINGER, H. (1926) Über den Hydrops Articulorum Intermittens und seine familiäre Form. Deutsche Medizinische Wochenschrift, 39, 68.

Wassmann, K. (1950) Palindromic rheumatism. Acta Medica Scandinavica, 139, 56.

Weber, F.P. (1946) Palindromic rheumatism. Lancet, ii, 931.

Weiner, A.D. \& GHORMLeY, R.K. (1956) Periodic benign synovitis: Idiopathic intermittent hydrarthrosis. Journal of Bone and Joint Surgery, 38A, 1039. 